SLAC-PUB-6412R

CU-TP-617 \& UCD-93-36

January 1994

$(\mathrm{T} / \mathrm{E})$

\title{
DIFFRACTIVE LEPTOPRODUCTION OF VECTOR MESONS IN QCD*
}

\author{
Stanley J. Brodsky \\ Stanford Linear Accelerator Center \\ Stanford University, Stanford, California 94309 \\ and \\ L. FRANKFURT ${ }^{\dagger}$ \\ School of Physics and Astronomy \\ Raymond and Beverly Sackler Faculty of Exact Sciences \\ Tel Aviv University, Ramat Aviv 69978, Israel \\ and \\ J. F. GUNION \\ Davis Institute for High Energy Physics \\ Department of Physics, University of California \\ Davis, California 95616 \\ and
}

A. H. MUELleR

Department of Physics, Columbia University, New York, New York 10027

and

\author{
M. STRIKMAN \\ Department of Physics, Pennsylvania State \\ University, University Park, Pennsylvania 16802
}

\footnotetext{
* Work supported by the Department of Energy, contracts DE-AC03-76SF00515, DE-FG02-93ER40771, and DE-FG03-91ER40674, Binational Science Foundation Grant 9200125, and Texas National Research Laboratory grant RGFY93-330.

$\dagger$ On leave of absence from the St. Petersburg Nuclear Physics Institute, Russia.

$\ddagger$ Also at the St. Petersburg Nuclear Physics Institute, Russia.
} 


\begin{abstract}
We demonstrate that the distinctive features of the forward differential cross section of diffractive leptoproduction of a vector meson can be legitimately calculated in perturbative QCD in terms of the light-cone $q \bar{q}$ wave function of the vector meson and the gluon distribution of the target. In particular, we calculate the $Q^{2}$ and nuclear dependence of the diffractive leptoproduction of vector mesons and estimate the cross section. The production of longitudinally polarized vector mesons by longitudinally polarized virtual photons is predicted to be the dominant component, yielding a cross section behaving as $Q^{-6}$. The nuclear dependence of the diffractive cross sections, which follows from a factorization theorem in perturbative QCD, provides important tests of color transparency as well as constraints on the shadowing of the gluon structure functions and the longitudinal structure functions of nuclei.
\end{abstract}

Submitted to Physical Review D. 


\section{Introduction}

We shall consider in this paper the small momentum transfer coherent electroproduction of vector mesons, $\gamma^{*}(q)+p \rightarrow V(q+\Delta)+(p-\Delta)$, where the target, $p$, can be either a nucleon or a nucleus, and where the state $(p-\Delta)$ is identical to $p$ except for a small momentum transfer. Here $V$ can be any possible vector meson, $\rho^{0}, \omega, \phi, J / \psi, \Upsilon$. We shall be concerned with the kinematic region where $x=Q^{2} / s$, and $M_{V}^{2} / s$ are small while $Q^{2} / \Lambda_{Q C D}^{2}$ is large. Small $x$ means that large longitudinal distances, in the rest frame of the target, are involved. The effective longitudinal distance during which the process takes place is large: $\Delta z \approx \frac{1}{M} \frac{s}{Q^{2}}[1]$, where $M$ is the target mass and $s=(p+q)^{2}$. Our interest is in the possibility of applying perturbative QCD (PQCD) to the calculation of hard processes characterized by large longitudinal distances. We demonstrate an interesting interplay of perturbative and nonperturbative QCD effects in the region where the coupling constant is small but distances are large which leads to a new way to probe light-cone wave functions of hadrons. In the case where $\Delta z \gg 2 R$, with $R$ the radius of a nuclear target, we predict that interesting color transparency effects will occur in diffractive electroproduction of vector mesons. In the last section of the paper, we briefly discuss QCD predictions for the nuclear dependence of the diffractive cross sections and show how such measurements can provide important constraints on the shadowing of the gluon structure functions and of the deep-inelastic longitudinal structure functions of nuclei.

In general, the physics underlying our PQCD calculation can be directly tested through the striking nuclear effects predicted in vector meson leptoproduction which differ from those that would result within the Glauber approximation. However, the thrust of the present paper is not a detailed discussion of nuclear effects, 
but rather a general description of the $Q^{2}$-dependence of the forward differential cross section, an analysis of the rigor of the PQCD calculation, a semi-quantitative estimate of the magnitude of the cross section, a prediction as to which polarization dominates the large $Q^{2}$ reaction, and a calculation of the nuclear dependence of electroproduction of vector mesons.

The main features of our perturbative QCD analysis agree with those obtained in the non-perturbative approach proposed by Landshoff and Donnachie [2] and discussed in more detail by Cudell [3]. In the DL model, the Pomeron is represented by the effective exchange of two non-perturbative gluons coupling via an effective constant coupling and the $\rho$ wavefunction is approximated as a non-relativistic vertex where the quark and anti-quark have equal four-momenta. In our analysis, the two gluon aspect of the QCD Pomeron emerges automatically at large $Q^{2}$ in a form directly related to the proton's gluon structure function. In addition we treat the relativistic structure of the vector meson generally. We find that the leading twist contribution to $\rho$ leptoproduction is controlled by the $\rho$ distribution amplitude $\phi(z, Q)$, the valence $q \bar{q}$ wavefunction which controls large momentum transfer exclusive processes. As in the DL model, PQCD predicts that the dominant leptoproduction amplitude couples a longitudinal photon to a longitudinally polarized vector meson, and the leading cross section $\sigma_{L}\left(s, Q^{2}\right)$ falls as $1 / Q^{6}$. The agreement of the data with this form is shown in Cudell's paper.[3]

We also predict that the cross section for production of a transversely polarized vector meson will fall as $Q^{-8}$. In fact, it is the end-point contributions (which complicate the analysis of elastic processes) that yield the dominant contribution to the cross section for a transversely polarized $V$. In contrast, we show that end-point configurations are unimportant for the diffractive production of longitudinally po- 
larized vector mesons.

Our work is also closely related to that of Ryskin [4] who has made detailed calculations of $J / \psi$ electroproduction in leading-logarithm in PQCD, employing the constituent quark model. In this work we focus on the dominant longitudinally polarized channels and find that an analogous analysis can be applied to light vector mesons. However, if in our formulae we take a nonrelativistic approximation for the wave function of the vector meson, then we find that the cross section for the production of the $J / \psi$ at large $Q^{2}$ is a factor of 4 less than that calculated in Ref. [4] in the nonrelativistic approximation. Unlike the heavy quarkonium case where the amplitude is controlled by the wavefunction at the origin and the lepton pair decay constant, light hadron leptoproduction probes the shape of the minimal Fock-state wavefunctions, i.e. the hadron distribution amplitudes $\phi_{V}(z, Q)$ as defined in Ref. [5]. This dependence allows tests of non-perturbative predictions from QCD sum rules and lattice gauge theory. We also find that this sensitivity may help explain the pattern of $S U(3)$-flavor symmetry breaking seen in the leptoproduction data.

The electroproduction of vector mesons has also been recently discussed by Kopeliovich et al. [6] (KNNZ) in the context of their constituent quark model approach to high energy, small momentum transfer processes in which the constituent quarks interact perturbatively with the nucleon. Indeed, there is much in common between the KNNZ approach and the present discussion, including their use of Eq. (20) for a nucleon target as derived and applied in Refs. [7] and [8]. However, the application of the nonrelativistic quark model to hard processes is questionable for this application, since in QCD hard processes should be calculated through the distribution of current, not constituent quarks [5]. Moreover, we find that the application of PQCD is only legitimate for the production of longi- 
tudinally polarized vector mesons. Thus, in distinction to Ref. [6], we expect a different nuclear dependence for the production of transversely and longitudinally polarized vector mesons. Further, the eikonal approximation used by KNNZ for the nuclear case is at variance with Eqs. (33) and (37), which are derived from factorization in PQCD. As a result we expect that nuclear effects will be leading twist - logarithmically decreasing at large $Q^{2}$ and increasing at small $x$. In contrast, KNNZ suggest, on the basis of their constituent quark model, that the nuclear effects in diffractive leptoproduction are a higher twist effect.

In order to achieve a simple result for the forward differential cross section, see e.g. Eq. (34), we find it necessary to work in the leading $\ln 1 / x$ and the leading ln $Q^{2} / \Lambda_{Q C D}^{2}$ limit. As discussed in the body of the paper we believe that it is also possible to calculate $d \sigma / d t$ at small $t$ in the leading $\ell n 1 / x$ approximation in terms of the unintegrated gluon distribution discussed sometime ago by Catani, Ciafaloni and Hautmann [9], without using the leading $\ln Q^{2} / \Lambda_{Q C D}^{2}$ approximation. However, such a calculation is not likely to lead to such a simple result as Eq. (34), which follows in the leading-double-logarithmic approximation. Thus, we have not pursued the single-logarithmic calculation, although it is clearly interesting to do so. At the same time we will explain at the end of Section 2.2 that corrections to the expression we obtain are numerically small.

Our final results should be taken with some caution. Since they are based on a leading-logarithmic, even leading-double-logarithmic, calculation, the normalizations may not be completely reliable. However, the $Q^{-6}$ dependence, the dominance of longitudinal polarization for both the virtual photon and the produced vector meson, and the proportionality of the cross section to the unintegrated gluon distribution in the target, and therefore the nontrivial dependence of cross 
section on atomic number, are firm predictions which should not depend on our logarithmic approximations.

\section{The Diffractive Cross Section in QCD Near $t=0$.}

In this section the near-forward differential cross section for $\gamma^{*}(q)+p \rightarrow V(q+$ $\Delta)+(p-\Delta)$ will be calculated in QCD. The target, labelled by its momentum $p$, is scattered into a state of momentum $p-\Delta$ which we assume to be a particle of the same species as $p$. For example, $p$ and $p-\Delta$ may both refer to protons. $V$ is a vector meson of mass $m_{V}$. We assume $s / m_{V}^{2} \gg 1, s / Q^{2} \gg 1$ and $-t \equiv-\Delta^{2} \ll Q^{2}$, and $s=(p+q)^{2}, q^{2}=-Q^{2}$. We also suppose $Q^{2} / \Lambda_{Q C D}^{2}$, and $Q^{2} / m_{V}^{2}$ are both much greater than one.

The differential cross section for the process described above is

$$
\frac{d \sigma_{\lambda \lambda^{\prime}}}{d t}=\frac{1}{16 \pi s^{2}}\left|\mathcal{M}_{\lambda \lambda^{\prime}}\right|^{2}
$$

where $\lambda$ is the polarization of the virtual photon and $\lambda^{\prime}$ is the polarization of the final state vector meson. In the large $s$ and large $Q^{2}$ limit, but with $s / Q^{2} \gg 1$, we expect the amplitude $\mathcal{M}$ to be dominated by two-gluon exchange, a particular graph of which is illustrated in Fig. 1. We will prove this statement for the production of longitudinally polarized vector meson. In Fig. 1, lines $k^{\prime}$ and $q-k^{\prime}$ are the quark and antiquark making up the vector meson. 


\subsection{Setting up the Calculation of the Matrix Element}

In proceeding to calculate $\mathcal{M}$ it is useful to view the process in a physical way. To that end, we choose a frame where $p$ is essentially at rest $\left(i . e ., p_{+} \ll q_{+}\right)$and where

$$
q=\left(q_{+}, q_{-}, q_{\perp}\right)=\left(q_{+}, \frac{-Q^{2}}{q_{+}}, 0\right)
$$

with $q_{+}=q_{0}+q_{3}, q_{-}=q_{0}-q_{3}$. Then $(q+\Delta)^{2}=m_{V}^{2}$ and $(p-\Delta)^{2}=M^{2}$ give

$$
\Delta_{-} \approx \frac{\left(Q^{2}+m_{V}^{2}+\Delta_{\perp}^{2}\right)}{q_{+}}, \quad \Delta_{+} \approx-p_{+} \frac{\Delta_{\perp}^{2}}{M^{2}}
$$

The polarization vectors are

$$
\epsilon^{\gamma}(\perp)=\left(0,0, \epsilon_{\perp}^{\gamma}\right), \epsilon^{\gamma}(L)=\left(\frac{q_{+}}{Q}, \frac{Q}{q_{+}}, 0_{\perp}\right)
$$

for the virtual photon and

$$
\epsilon^{V}(\perp)=\left(0,0, \epsilon_{\perp}^{V}\right), \epsilon^{V}(L)=\left(\frac{\left(q_{+}+\Delta_{+}\right)}{m_{V}}, \frac{-m_{V}}{\left(q_{+}+\Delta_{+}\right)}, 0_{\perp}\right)
$$

where we have dropped terms proportional to $\Delta_{\perp}$ in $(2.5)$.

The process illustrated in Fig. 1 takes place, sequentially in time, as follows.

(i) The virtual photon breaks up into a quark-antiquark pair with a lifetime $\tau_{i}$ given by

$$
q_{+} \tau_{i}^{-1}=Q^{2}+\frac{k_{\perp}^{2}+m^{2}}{z(1-z)} \approx Q^{2}
$$

Here $m$ is the current quark mass. This estimate is valid for the production of a longitudinally polarized vector meson only. In the case of a transversely 
polarized vector meson, the end-point non-perturbative contribution arises from the kinematical region where $z$ is close to 0 or $1: z, 1-z \sim m^{2} / Q^{2}$ as in the aligned-jet model of Ref. [10] adapted to QCD in Ref. [11]. (See also the discussion below.)

(ii) The quark-antiquark pair then scatters off the target proton.

(iii) The quark-antiquark pair then lives a time $\tau_{f}$ determined by

$$
q_{+} \tau_{f}^{-1}=\frac{k_{\perp}^{2}+m^{2}}{z(1-z)}
$$

before the final state vector meson is formed. We note that $\tau_{f} \geq \tau_{i}$.

Thus, the amplitude $\mathcal{M}$ can be written as a product of three factors: (i) the wavefunction giving the amplitude for the virtual photon to break into a quarkantiquark pair; (ii) the scattering amplitude of the quark-antiquark pair on the target; and (iii) the wavefunction giving the amplitude for the scattered quarkantiquark pair of flavor $f$ to become a vector meson. Following the conventions of Ref. [5], we have

$$
\begin{aligned}
\mathcal{M}_{f}= & \sqrt{N_{c}} \sum_{\lambda_{1}, \lambda_{2}} \int \frac{d^{2} k_{\perp} d^{2} k_{\perp}^{\prime}}{\left(16 \pi^{3}\right)^{2}} \int_{0}^{1} d z \int_{0}^{1} d z^{\prime} \\
& \times \psi_{\lambda_{1} \lambda_{2}}^{V *}\left(k_{\perp}^{\prime}, z^{\prime}\right) T_{\lambda_{1} \lambda_{2}}\left(k_{\perp}^{\prime}, z^{\prime} ; k_{\perp}, z\right) \psi_{\lambda_{1} \lambda_{2}}^{\gamma}\left(k_{\perp}, z\right),
\end{aligned}
$$

where $\lambda_{1}$ and $\lambda_{2}$ are the helicities of the quark-antiquark pair which are conserved during the scattering off the target. $\psi^{V}$ and $\psi^{\gamma}$ are the light-cone wavefunctions in the notation of Ref. [5]. We have explicitly extracted the sum over the $N_{c}$ colors of the quarks and the $1 / \sqrt{N_{c}}$ from the color singlet normalization of $\psi^{V}$. Thus, $\psi^{V}$ and $T$ correspond to the wave function and scattering amplitude for a quark-antiquark pair of definite color. 
We only obtain a simple result, in terms of the gluon distribution of the proton, in the leading-logarithmic approximation (in longitudinal and transverse momentum) for $T$. In this leading-logarithmic approximation, the time of scattering of the quark-antiquark pair with the target is much less than $\tau_{i}$ so that $T$ is effectively given as the on-shell scattering of a quark-antiquark pair off the target. Our task now is to evaluate $\psi^{\gamma}$ and $T$ and thus to express the amplitude, $\mathcal{M}_{f}$, in terms of integrals over the exclusive wavefunction $\psi^{V}$.

\section{2. $\psi^{\gamma}$ And the Scattering of the Quark-Antiquark Pair By the} TARGET

The evaluation of $\psi^{\gamma}$ at lowest order in $e$ is straightforward. In the convention of Ref. [5],

$$
\psi_{\lambda_{1} \lambda_{2}}^{\gamma}\left(k_{\perp}, z\right)=e e_{f} \frac{\bar{u}_{\lambda_{1}}(k) \gamma \cdot \epsilon^{\gamma} v_{\lambda_{2}}(q-k)}{\sqrt{k_{+}}\left(q_{-}-\frac{k_{\perp}^{2}+m^{2}}{k_{+}}-\frac{k_{\perp}^{2}+m^{2}}{(q-k)_{+}}\right) \sqrt{(q-k)_{+}}}
$$

which leads to

$$
\psi_{\lambda_{1} \lambda_{2}}^{\gamma}\left(k_{\perp}, z\right)=-e e_{f} \frac{\bar{u}_{\lambda_{1}}(k) \gamma \cdot \epsilon^{\gamma} v_{\lambda_{2}}(q-k)}{\sqrt{z(1-z)}\left(Q^{2}+\frac{k_{\perp}^{2}+m^{2}}{z(1-z)}\right)}
$$

In Eq. (2.10), $e$ is the charge of the proton and $e_{f}$ is the charge of a quark of flavor $f$, as a fraction of the proton's charge. We omit the label $f$ on $\psi^{\gamma}$ for simplicity of notation. We see from (2.4) that longitudinal polarization apparently gives the dominant contribution, though we must wait until we have calculated $\psi^{V}$ to see that this is indeed the case. Anticipating this result we set $\epsilon^{\gamma}=\epsilon^{\gamma}(L)$ and, using 
$(2.4)$, obtain

$$
\psi_{\lambda_{1} \lambda_{2}}^{\gamma}\left(k_{\perp}, z\right)=-\frac{e e_{f} Q \delta_{\lambda_{1},-\lambda_{2}}}{Q^{2}+\frac{k_{\perp}^{2}+m^{2}}{z(1-z)}}
$$

Now consider the scattering of the quark-antiquark pair by the target. The relevant light-cone perturbation theory graphs are shown in Fig. 2. (It is important to note our convention of using the momenta $q-k^{\prime}$ and $k^{\prime}+\Delta$ that enter $\psi^{V}$ to define the momenta of the lines.) As we have mentioned before, a simple result emerges only in the leading-logarithmic approximation in $\ell n Q^{2} / s$. In that case, $\ell_{+} / k_{+} \ll 1$ and the dominant couplings of the lines $\ell$ and $\ell+\Delta$ to the quarkantiquark pair occur with a $\gamma_{+}$(in a covariant gauge). Thus, the vertices of the lines $\ell+\Delta$ and $\ell$ with the quark-antiquark pair are exactly the same for each of the graphs in Fig. 2. Further, all energy denominators are dominated by the $\ell$ and $\ell+\Delta$ lines so that the energy denominators are also the same for each of the graphs in Fig. 2. The differences between the different graphs in Fig. 2 are only in the labelling of the momenta on the left-hand side of the diagram as they emerge from the $\psi^{\gamma}$ wavefunction. The result obtained in the $\Delta_{\perp} \ll \ell_{\perp}$ limit being considered is very simple:

$$
\begin{aligned}
& T_{\lambda_{1} \lambda_{2}}\left(k_{\perp}^{\prime}, z^{\prime} ; k_{\perp}, z\right)= \\
& \quad \times 16 \pi^{3} \int\left\{2 \delta\left(k_{\perp}^{\prime}-k_{\perp}\right)-\delta\left(k_{\perp}^{\prime}-k_{\perp}+\ell_{\perp}\right)-\delta\left(k_{\perp}^{\prime}-k_{\perp}-\ell_{\perp}\right)\right\} \\
& \quad \times \delta\left(z^{\prime}-z\right) \mathcal{I}(\ell) \frac{d^{2} \ell_{\perp} d \ell_{+}}{16 \pi^{3}}
\end{aligned}
$$

where $\mathcal{I}(\ell)$ represents the gluon propagators and zero-angle gluon-nucleon scattering amplitude shown in Fig. 1.

Before attempting to evaluate the $d^{2} \ell_{\perp} d \ell_{+}$integral involving $\mathcal{I}$, let us first do the integrals over $d^{2} k_{\perp}^{\prime} d z^{\prime} / 16 \pi^{3}$ indicated in (2.8). Using (2.12) one arrives at the 
combination

$$
2 \psi_{\lambda_{1} \lambda_{2}}^{\gamma}\left(k_{\perp}^{\prime}, z\right)-\psi_{\lambda_{1} \lambda_{2}}^{\gamma}\left(k_{\perp}^{\prime}+\ell_{\perp}, z\right)-\psi_{\lambda_{1} \lambda_{2}}^{\gamma}\left(k_{\perp}^{\prime}-\ell_{\perp}, z\right)=\Delta \psi_{\lambda_{1} \lambda_{2}}^{\gamma}
$$

which, using (2.11), gives

$$
\Delta \psi_{\lambda_{1} \lambda_{2}}^{\gamma}=\frac{-2 e e_{f} \delta_{\lambda_{1},-\lambda_{2}} Q \ell_{\perp}^{2}}{\left[Q^{2}+\frac{k_{\perp}^{2}+m^{2}}{z(1-z)}\right]^{2} z(1-z)}
$$

when $\ell_{\perp}^{2} / Q^{2} \ll 1$. Thus (relabelling $k_{\perp}^{\prime} \rightarrow k_{\perp}$ ),

$$
\mathcal{M}_{f}=\sqrt{N_{c}} \Sigma_{\lambda_{1}, \lambda_{2}} \int \frac{d^{2} k_{\perp}}{16 \pi^{3}} \int_{0}^{1} d z \psi_{\lambda_{1} \lambda_{2}}^{V *}\left(k_{\perp}, z\right) \mathcal{I}(\ell) \frac{d^{2} \ell_{\perp} d \ell_{+}}{16 \pi^{3}} \Delta \psi_{\lambda_{1} \lambda_{2}}^{\gamma}
$$

If instead of a final state vector meson we were considering a virtual photon identical to the initial state photon, the exact same dependence on $\ell_{\perp}$ and $\ell_{+}$would appear. This allows one to identify an integral over $\mathcal{I}(\ell)$ with the gluon distribution. Indeed, in the leading-logarithmic approximation in $\ell n 1 / x$ and $\ell n Q^{2} / \Lambda^{2}$, where $\mathcal{I}$ is purely imaginary,

$$
\int \frac{d^{2} \ell_{\perp} d \ell_{+}}{16 \pi^{3}} \ell_{\perp}^{2} \mathcal{I}(\ell)=i \frac{4 \pi^{2} T_{R} \alpha_{s}}{N_{c}}\left(s+Q^{2}\right) x G\left(x, Q^{2}\right)
$$

where the factor of $T_{R} / N_{c}$ arises by virtue of averaging over the color and matching anti-color of the initial quark and antiquark. (In the usual convention $T_{R}=\frac{1}{2}$.) The simplest way to determine the normalizing factor in Eq. (2.16) is to compare the integral on the left hand side of (2.16) with the known relation of the longitudinal structure function with the gluon density. Once one is sure that the gluon distribution should emerge from the integration on the left-hand side of (2.16), the 
normalization is most easily set by taking the target to be a quark and calculating the lowest order contribution to $\mathcal{I}$. However, to establish that $x=Q^{2} / s$ and $Q^{2}$ are the appropriate arguments in $x G\left(x, Q^{2}\right)$ requires employing the leading $\ell n 1 / x$ and $\ln Q^{2} / \Lambda_{Q C D}^{2}$ approximations.

We can understand these values from the simple picture on which Eqs. (2.8) and (2.15) are based. Namely, we must have a strict sequence of events in which (i) the virtual photon breaks up into an approximately on-shell quark-antiquark pair; followed by (ii) the scattering of the on-shell quark-antiquark pair by the target; and, finally, (iii) the scattered quark-antiquark pair turns into the vector meson. This picture requires that the time of scattering of the quark-antiquark pair must be much less than $\tau_{i}$ and $\tau_{f}$ defined earlier: i.e. $\tau \ll \tau_{i} \leq \tau_{f}$. Using (recall that we employ OFPT here)

$$
\tau \sim \frac{1}{\ell_{-}}=\frac{\ell_{+}}{\ell_{\perp}^{2}}
$$

this condition reduces to

$$
\ell_{+} \ll \frac{q_{+} \ell_{\perp}^{2}}{Q^{2}}
$$

Meanwhile, the dominance of the imaginary part implies that the - components of the four-momenta are approximately conserved from the initial to the intermediate state where the $p+\ell$ line is cut, leading to $(p+\ell)_{-} \simeq p_{-}$(neglecting - components of order $\left.1 / q_{+}\right)$. With this, we compute $\hat{s}=(p+\ell)^{2} \simeq M^{2}+p_{-} \ell_{+} \ll \ell_{\perp}^{2} s / Q^{2}$, with the last inequality coming from employing (2.18). In the leading $\ln 1 / x$ approximation we allow integration over the parton-gluon scattering subprocess energy, $\hat{s}$, up to this upper limit. Recalling that the $x_{g}$ argument of $G\left(x_{g}, Q_{g}^{2}\right)$ is set by $x_{g} \hat{s} \sim \ell_{\perp}^{2}$, we see that in the leading $\ell n 1 / x$ approximation $x_{g}$ should be 
identified with $x=Q^{2} / s$. To determine the appropriate argument $Q_{g}^{2}$, we note that the simple form for $\Delta \psi^{\gamma}$ given in (2.14) depends on

$$
\frac{\ell_{\perp}^{2}}{Q^{2}} \ll 1
$$

In leading $\ell n Q^{2} / \Lambda_{Q C D}^{2}$ we integrate $\ell_{\perp}^{2}$ up to the maximum allowed by this relation, and it is this maximum which determines the argument $Q_{g}^{2}$ appearing in $G$. The result is obviously $Q_{g}^{2} \sim Q^{2}$. (We note here that so long as $\Delta_{\perp}^{2} / \Lambda_{Q C D}^{2} \ll 1$, the left-hand side of Eq. (2.16) does not depend on $\Delta$. From (2.3), we see that $\Delta_{-}$is comparable to $\ell_{-}=\ell_{\perp}^{2} / \ell_{+}$only when $\ell_{\perp}^{2} / \ell_{+}$reaches its smallest value, $Q^{2} / q_{+} \approx \Delta_{-}$.) It is useful to note that these conclusions match closely those obtained in Ref. [7] where the scattering of a quark-antiquark pair off a target is given by

$$
\sigma\left(b^{2}\right)=\frac{2 \pi^{2}}{3}\left(b^{2} \alpha_{s}\left(Q^{2}\right) \bar{x} G_{N}\left(\bar{x}, Q^{2}\right)\right)_{\bar{x}=1 / s b^{2}, Q^{2}=1 / b^{2}}
$$

where, for simplicity, the result has been stated for the case $x_{q} \sim x_{\bar{q}} \sim 1 / 2$.

Can one do better than the leading double log approximation? The answer should be yes. One should be able to eliminate the restriction (2.19) and derive an expression for $\mathcal{M}$ in terms of integrals over the unintegrated gluon distribution and the vector meson exclusive wavefunction. The result, however, will be significantly more complicated than the answer we are about to give for the leading doublelogarithmic approximation. At the same time, the corrections to (2.16) resulting from the elimination of the restriction (2.19) are numerically small. A simple way to justify this statement is to perform the calculation in impact parameter space where non- $\ell n Q^{2} / \Lambda_{Q C D}^{2}$ corrections arise from the decomposition of the matrix element of the real part of $e^{i \ell_{\perp} \cdot b_{\perp}}-1$ between the wave functions of the virtual 
photon $\gamma^{*}$ and a vector meson in impact parameter space. Here $b_{\perp}$ is the interquark distance in the meson wavefunction and $\ell_{\perp}$ is the transverse momentum of the gluon shown in Fig. 2. The second order term in $\left(\ell_{\perp} \cdot b_{\perp}\right)$ leads to Eq. (2.15). The corrections in question arise starting at the fourth order in the expansion contain a factor of $1 / 4$ ! and are therefore small.

\subsection{The Final State Vector Meson Wavefunction}

Using (2.16) and (2.14) in (2.15), and $\left(s+Q^{2}\right) \simeq s$, one obtains

$$
\mathcal{M}_{f}=\frac{-8 i s \pi^{2} e e_{f} \alpha_{s}}{Q^{3}} \frac{T_{R}}{\sqrt{N_{c}}} x G\left(x, Q^{2}\right) \int \frac{d^{2} k_{\perp}}{16 \pi^{3}} \int_{0}^{1} \frac{d z}{z(1-z)} \Sigma_{\lambda} \psi_{\lambda-\lambda}^{V}\left(k_{\perp}, z\right)
$$

where

$$
\psi_{\lambda-\lambda}^{V}\left(k_{\perp}, z\right)=N_{V} \frac{\bar{v}_{-\lambda}(q-k)}{\sqrt{1-z}} \gamma \cdot \epsilon^{V} \frac{u_{\lambda}(k)}{\sqrt{z}} \psi^{V}\left(k_{\perp}, z\right) \frac{1}{m_{V}} .
$$

Here, we have introduced a normalization factor $N_{V}$ for the particular spin state of $V$ being considered. (In the conventions of Ref. [5], $N_{V}=1 / \sqrt{2}$ for $\epsilon_{V}(L)$.)

We are now in a position to verify that longitudinal polarizations give the dominant contribution. From (2.10) and (2.22) it is straightforward to see that longitudinal polarizations give the leading contribution so long as $Q$ is much larger than all quark and vector meson masses. In this case, using (2.5) in (2.22) gives

$$
\psi_{\lambda-\lambda}^{V}\left(k_{\perp}, z\right)=-N_{V} \psi^{V}\left(k_{\perp}, z\right)
$$

which yields (after performing $\Sigma_{\lambda}$ and setting $T_{R}=1 / 2$ )

$$
\mathcal{M}_{f}=\frac{8 i s \pi^{2} e e_{f} \alpha}{Q^{3} \sqrt{N_{c}}} x G(x, Q) N_{V} \int_{0}^{1} \frac{d z}{z(1-z)} \phi^{V}(Q, z)
$$


where

$$
\phi^{V}(Q, z)=\int \frac{d^{2} k_{\perp}}{16 \pi^{3}} \psi^{V}\left(k_{\perp}, z\right)
$$

is the distribution amplitude for longitudinally polarized vector mesons with the restriction $k_{\perp}^{2}<Q^{2}$ understood for the integration in (2.25). In getting the correct normalization for the cross section, it will be important to note that this result does not yet include the flavor normalization for the $V$ wavefunction. (The spin normalization factor is contained in $N_{V}$.)

In order to obtain an absolute normalization for $\mathcal{M}_{f}$, it is necessary to relate $\phi^{V}$ to the experimental observable $f_{V}$ defined by

$$
\left\langle 0\left|J_{e . m .}^{\mu}\right| V\right\rangle=\frac{\sqrt{2} e f_{V}}{m_{V} \epsilon^{\mu}},
$$

in terms of which the decay width for $V \rightarrow e^{+} e^{-}$is given by

$$
\Gamma_{V}=\frac{8 \pi \alpha^{2} f_{V}^{2}}{3 m_{V}}
$$

We demonstrate in the Appendix that

$$
\int d z \phi^{V}(z)=\frac{f_{V}}{\sqrt{N_{c}} e_{f} 2 \sqrt{2} N_{V}} .
$$

Defining $\eta_{V}$ as

$$
\eta_{V} \equiv \frac{1}{2} \frac{\int \frac{d z}{z(1-z)} \phi^{V}(z)}{\int d z \phi^{V}(z)}
$$

we obtain

$$
\mathcal{M}_{f}=\frac{8 i s \pi^{2} f_{V} e \alpha_{s} \eta_{V}}{\sqrt{2} Q^{3} N_{c}} x G(x, Q)
$$

The parameter $\eta_{V}$ is the effective inverse moment of the vector meson distribution amplitude that controls the leading twist contribution to the leptoproduction 
amplitude. Higher particle number Fock state amplitudes such as the $q \bar{q} g$ in a physical gauge have a suppressed coupling to the small-size quark pair; in order to compensate their higher mass dimensions, they must be accompanied by further powers of $1 / Q$. Note that both $e_{f}$ and $N_{V}$ have cancelled out in relating $\mathcal{M}_{f}$ to the experimental observable $f_{V}$.

In order to estimate the cross section it is convenient to consider two extreme examples for the shape of the $\rho$ distribution amplitude:

$$
\begin{gathered}
\phi_{1}^{V}=\frac{3}{\sqrt{2} N_{V} \sqrt{N_{c}}} \frac{f_{V}}{e_{f}} z(1-z) \\
\phi_{2}^{V}=\frac{15}{\sqrt{2} N_{V} \sqrt{N_{c}}} \frac{f_{V}}{e_{f}} z(1-z)(1-2 z)^{2} .
\end{gathered}
$$

Here $\phi_{1}$ is the asymptotic form of the distribution amplitude, while $\phi_{2}$ is of the form suggested by Chernyak and Zhitnitsky [12] for pions. The normalizations of $\phi_{1}^{V}$ and $\phi_{2}^{V}$ are chosen for consistency with Eq. (2.28). A simple calculation gives $\eta_{V}=3$ for $\phi_{1}$ and $\eta_{V}=5$ for $\phi_{2}$. We emphasize that in QCD amplitudes for hard processes are expressed in terms of the minimal Fock component of the light-cone wave function of the meson (see e.g. Eq. (2.21)), not in terms of constituent quark model components. This is important when the physics of color screening, which is relevant to the transition from the non-perturbative to the perturbative regime, is accounted for ( $c f$. the discussion in Section 2.6 regarding the difference between the production of transverse and longitudinally polarized vector mesons, and the discussion of color transparency in Section 2.8). 


\subsection{The Flavor Dependence of the Cross Section}

Thus far, we have not discussed the flavor dependence of the wavefunction of the final state vector meson. If $V$ refers to a neutral $\rho$ meson, then the relevant wavefunction is $\frac{1}{\sqrt{2}}(|u \bar{u}>-| d \bar{d}>)$. This would imply a replacement of $e_{f}$ in our calculations of both $\mathcal{M}_{f}$ and $f_{V}$ by $e_{f} \rightarrow \frac{1}{\sqrt{2}}\left(e_{u}-e_{d}\right)=1 / \sqrt{2}$. However, since $e_{f}$ cancels out when $\mathcal{M}_{f}$ is expressed in terms of $f_{V}$, as in Eq. (2.30), the result is that we may use Eq. (2.30) for the total amplitude without change provided we employ the appropriate value for $f_{\rho}$. With our normalization conventions, $f_{\rho} \approx 107$ $\mathrm{MeV}$ for the $\rho^{0}$. If $V$ refers to a $J / \psi$ one can simply use $e_{f}=e_{c}=2 / 3$. Again, the explicit value of $e_{f}$ disappears if $\mathcal{M}_{f}$ is expressed in terms of $f_{J / \psi}$. However, for the $J / \psi$ the value of $\eta_{V}$ is expected to differ substantially from that for the $\rho$ since neither of the wavefunctions given in (2.31) and (2.32) is appropriate; a wavefunction having $z \approx 1 / 2$ would be more suitable.

\subsection{The Differential Cross Section}

We shall now write the differential cross section for $\rho^{0}$ production. Modifications for other lepto-produced neutral vector mesons are straightforward and involve choosing a distribution amplitude to replace (2.31) or (2.32). Using Eq. (2.30) in Eq. (2.1) gives

$$
\left.\frac{d \sigma}{d t}\right|_{t=0}\left(\gamma^{*} N \rightarrow V N\right)=\frac{8 \pi^{4} f_{V}^{2} \alpha_{E M} \alpha_{s}^{2}(Q) \eta_{V}^{2}[x G(x, Q)]^{2}}{Q^{6} N_{c}^{2}} .
$$

It is important to keep in mind that this result gives the differential cross section for a longitudinally polarized photon to produce a longitudinally polarized $\rho^{0}$; i.e. it is not spin-averaged over initial photon states. As an aside, we note that the 
$t$-dependence of this diffractive cross section is controlled by the quasi-local twogluon matrix element of the nucleon; in principle, it could have a different fall-off than the elastic form factors since the momentum transfer is shared by the two gluons.

It is convenient to rewrite Eq. (2.33) in terms of the leptonic width, $\Gamma_{V}$ using Eq. (2.27),

$$
\left.\frac{d \sigma}{d t}\right|_{t=0}\left(\gamma^{*} N \rightarrow V N\right)=\frac{3 \pi^{3} \Gamma_{V} m_{V} \alpha_{s}^{2}(Q) \eta_{V}^{2}[x G(x, Q)]^{2}}{\alpha_{E M} Q^{6} N_{c}^{2}}
$$

since the coherent sum over the contributing flavors is identical for the diffractive amplitude and the decay amplitude. Our prediction for the $J / \psi$ leptoproduction cross section using Eq. (2.34) is smaller than the one obtained in Ref. [4] by a factor of 4 , if we assume, as in Ref. [4], a nonrelativistic form $\sim \delta(z-1 / 2)$ for the $J / \psi$ meson wave function so that $\eta_{\psi} \approx 2$.

We can also use dispersion relations to determine the real part of the amplitude. For small enough $x$ and large $Q^{2}$ the contribution of the real part is not negligible since the effective QCD pomeron intercept is above 1. Including the real contribution as a perturbation we get

$$
\left.\frac{d \sigma_{\gamma^{*} N \rightarrow V N}^{L}}{d t}\right|_{t=0}=\frac{3 \pi^{3} \Gamma_{V} m_{V} \alpha_{s}^{2}(Q) \eta_{V}^{2}\left|\left(1+i \frac{\pi}{2} \frac{d}{d \ln x}\right) x G_{T}(x, Q)\right|^{2}}{\alpha_{E M} Q^{6} N_{c}^{2}}
$$

Since the gluon density rises rapidly at small $x$ in the large $Q^{2}$ domain, we predict a very substantial rise of the diffractive cross section with energy at large $Q^{2}$. For example, for $Q^{2} \sim 10 \mathrm{GeV}^{2}$, we predict a rise of the diffractive cross section at small $x$ by as much as a factor of 100 at HERA energies as compared to the 
cross section measured at CERN. Obviously this effect would also substantially modify the $Q^{2}$-dependence of the cross section at large energies.

Let us now compare Eq. (2.33) with experimental data for $\rho$-meson production at large $Q^{2}$ and small $x$. Such data are available from EMC [13] and NMC [14,15] and also from the E-665 FNAL experiment [16]. We will use the latest data of NMC [15] which were obtained at the highest $Q^{2}$ with special attention to removing backgrounds due to inelastic processes. We also note that the data of E-665 [16], which extend to somewhat smaller $Q^{2}$, are generally consistent with NMC data. All of these data confirm the important role of the longitudinal contribution at large $Q^{2}$ from the measurement of the polarization of the $\rho$. If $s$-channel helicity conservation is assumed in the transition $\gamma^{*} \rightarrow \rho$, this also determines the ratio of $\sigma_{L} / \sigma_{T}$

To convert the leptoproduction cross section $d^{2} \sigma / d Q^{2} d \nu$ from $\mu N$ to the virtual photoproduction cross section, we use the standard relation $\sigma_{\mu N}\left(Q^{2}, \nu\right)=$ $\Gamma \sigma_{\gamma^{*} N}\left(Q^{2}, \nu\right)$, where $\Gamma=\alpha_{E M}\left(\nu-Q^{2} /(2 M)\right) /\left(2 \pi Q^{2} E^{2}(1-\epsilon)\right)$ and $\sigma_{\gamma^{*} N}=$ $\sigma_{T}+\epsilon \sigma_{L}$. Using $\sigma_{t o t}\left(\gamma^{*} N \rightarrow \rho N\right)$ as determined by NMC from $\mu D$ data, the slope of the $t$-dependence of the cross section, $b=4.3 \pm 0.6 \pm 0.7 \mathrm{GeV}^{-2}$ as measured by NMC, and the NMC estimate for $\sigma_{i n c o h} / \sigma_{t o t}=0.55 \pm 0.08$ and of $\sigma_{\gamma^{*} N \rightarrow \rho^{0} N}^{L} / \sigma_{\gamma^{*} N \rightarrow \rho^{0} N}^{T} \sim 2.0$ at $Q^{2}=6 \mathrm{GeV}^{2}$ and $\epsilon=0.8$, we can estimate

$$
\left.\frac{d \sigma_{\gamma^{*} N \rightarrow \rho^{0} N}^{L \text { experiment }}}{d t}\right|_{t=0} \sim \frac{14-27 \mathrm{nb}}{\mathrm{GeV}^{2}}
$$

for $Q^{2}=10 \mathrm{GeV}^{2}$. (In deriving this range we also assumed that $\sigma_{L} / \sigma_{T}$ is either the same at $Q^{2}=10 \mathrm{GeV}^{2}$ as at $Q^{2}=6 \mathrm{GeV}^{2}$ or increases linearly above $Q^{2}=6 \mathrm{GeV}^{2}$ as $\sim Q^{2}$.) 
Let us now compare this result with the leading-logarithmic prediction of Eq. (2.33). The HMRS $D 0^{\prime}$ parameterization of the gluon distribution in the proton $[17]$ gives the value $\alpha_{s}(Q)[x G(x, Q)]=0.67$ at the NMC kinematics $x \simeq 0.06$, $Q^{2}=10 \mathrm{GeV}^{2}$. The sensitivity of the parameterization to uncalculated higher order terms and the uncertainty in the evolution scale is illustrated by noting that $\alpha_{s}[x G]=0.76$ if we use $\frac{1}{2} Q^{2}$ instead of $Q^{2}$ for the arguments of $\alpha_{s}$ and $G$. Thus we obtain a range of predictions

$$
\left.\frac{d \sigma_{\gamma^{*} N \rightarrow \rho^{0} N}^{L}}{d t}\right|_{t=0} \sim \frac{(13-17)-(36-47) \mathrm{nb}}{\mathrm{GeV}^{2}}
$$

The lower range corresponds to $\eta_{\rho}=3$ assuming the asymptotic form of the $\rho_{L}$ distribution amplitude $\sim z(1-z)$. The upper range corresponds to $\eta_{\rho}=5$ assuming that the $\rho_{L}$ distribution amplitude is similar to the $\mathrm{CZ}$ wave function of a pion. The distribution amplitude suggested by Chernyak and Zhitnitski [12] from QCD sum rules for the $\rho_{L}$ actually corresponds to a narrower quark distribution than for the pion, but it is still broader than the asymptotic form. Thus $\eta_{\rho_{L}}^{C Z} \sim 3.3-3.5$. If we trust our leading-order estimates, then this value of $\eta_{\rho_{L}}$ leads to

$$
\left.\frac{d \sigma_{\gamma^{*} N \rightarrow \rho^{0} N}^{L}}{d t}\right|_{t=0, \eta_{\rho_{L}}^{C Z}} \sim \frac{16-23 \mathrm{nb}}{\mathrm{GeV}^{2}}
$$

which is close to the empirical diffractive $\rho$ leptoproduction cross section. More generally, $\rho$ electroproduction will allow us to test determinations of the meson distribution amplitudes from lattice gauge theory or other non-perturbative QCD computations.

Equation (2.34) also allows us to predict the ratio of the yields of various vector 
mesons at $Q^{2} \gg m_{V}^{2}$ :

$$
\left.R\left(V_{1}, V_{2}\right) \equiv \frac{\frac{d \sigma\left(\gamma_{L}+T \rightarrow V_{1}+T\right)}{d t}}{\frac{d \sigma\left(\gamma_{L}+T \rightarrow V_{2}+T\right)}{d t}}\right|_{t=0}=\frac{\Gamma_{V_{1}} m_{V_{1}}}{\Gamma_{V_{2}} m_{V_{2}}} \frac{\eta_{V_{1}}^{2}}{\eta_{V_{2}}^{2}}
$$

It can be seen from Eq. (2.35) that we predict $R(\phi, \rho) \sim 1.0 \times e_{f}^{2}(\phi) / e_{f}^{2}(\rho)$ for $\eta_{\phi} / \eta_{\rho} \sim 0.9$ suggested by CZ [12]. We also find $R(J / \psi, \rho) \sim 1.2 \times e_{f}^{2}(J / \Psi) / e_{f}^{2}(\rho)$ for $\eta_{J / \psi}=2$ corresponding to $\phi_{J / \psi} \sim \delta(z-1 / 2)$. Here, we have extracted from these two $R$ results the ratios of the effective charge squared, where $e_{f}^{2}(V)=$ $1 / 2,1 / 18,1 / 9,4 / 9$ for the $\rho, \omega, \phi, \Psi$, respectively (for standard $\mathrm{SU}(3)$ wave functions), which might naively be expected to determine the $R$ 's.

Another interesting feature of the QCD prediction for the production of a vector meson at large $Q^{2}$ is the universality of the $t$-dependence of the process - it is determined by a universal two-gluon form factor, independent of the vector meson type. The upper part of the amplitude corresponding to the transition $\gamma^{*} \rightarrow V$ is effectively dipole-like at large $Q^{2}$; i.e. it should depend weakly on $t$ so long as $-t \ll Q^{2}$, implying that the $t$-dependence of the leptoproduction cross section primarily reflects the $t$-dependence of the gluon - nucleon scattering amplitude. The slope of this $t$-dependence should increase slowly with the incident energy due to the Gribov diffusion - shrinkage of the diffractive cone. The data on exclusive production of vector mesons at high energies supports this prediction - the large $Q^{2} \rho$-meson leptoproduction cross section has a slope $d \sigma / d t \propto e^{b t}$ corresponding to $b=4.3 \mathrm{GeV}^{-2}[14]$, which is similar to the small slope $b \sim 3.5-5.5 \mathrm{GeV}^{-2}$ observed for exclusive $J / \psi$-meson photoproduction. 


\subsection{Non-Perturbative QCD Effects}

The $Q^{2}$ dependence of the diffractive vector meson leptoproduction amplitude in the PQCD analysis reflects the overlapping integral between the light cone wave functions of $\gamma^{*}$ and the vector meson. Since the wave function of $\gamma_{L}^{*}$ is $\propto z$ or $(1-z)$ when $z$ or $1-z$ vanish, the end-point contributions to the cross section for the production of longitudinally polarized vector mesons, arising from Eq. (2.8), are small and of order $\sim Q^{2} / m_{V}^{2}\left(Q^{2}\right)^{6}$. Since the wave function of a vector meson bound state should be less singular at $z \sim 0$ and 1 than the wave function of the $\gamma^{*}$, we conclude that for $\sigma_{L}$ the end-point contribution may be neglected. Thus the longitudinal cross section can be safely calculated in terms of PQCD.

The situation is the opposite in the case of the production of a vector meson by a photon with transverse polarization. The wave function of a transversely polarized photon is constant at $z \sim 0$ and 1. As a result, in this case the endpoint contribution is enhanced. To demonstrate the importance of the end-point contribution, let us consider the process where a final state photon is produced instead of a vector meson; i.e. the process:

$$
\gamma^{*}+T \rightarrow \gamma+T .
$$

For this reaction, the end-point contribution leads to the cross section

$$
\left.\frac{d \sigma^{\gamma^{*}+T \rightarrow \gamma+T}}{d t}\right|_{t=0} \sim \frac{1}{Q^{4}} .
$$

Now note that the wave function of a transversely polarized vector meson is less singular than that of the photon as $z \rightarrow 0,1$. In fact, PQCD predicts that the $q \bar{q}$ component decreases at least as fast as $\propto z$ or $(1-z)$ when $z$ or $1-z$ are 
small. Consequently, the cross section for electroproduction of transversely polarized svector mesons should fall at least as fast as $1 / Q^{8}$. (This contribution is additionally suppressed by a double logarithmic Sudakov-type form factor.) A similar dependence of the cross section arises from other kinematical regions. Thus the experimental investigation of the ratio of longitudinally and transverse polarized vector mesons would help to clarify the relative roles of non-perturbative QCD-end-point contributions and hard physics.

\subsection{Diffractive Leptoproduction on NuClei}

A key feature of the predictions of PQCD for forward diffractive vector meson leptoproduction with $1 / 2 m_{N} x \gg 2 R_{A}$ is the dominance of small size wavefunction configurations. The fact that the integration range of $k_{\perp}^{2}$ in the vector meson light-cone wavefunction in Eq. (2.25) extends to $\sim Q^{2}$ implies that the important $q \bar{q}$ configurations coupling to the virtual photon have transverse separations $b_{\perp} \sim$ $1 / Q$. Thus, even in a nuclear target, color screening implies that the coherent $q \bar{q}$ system can only weakly interact, and in leading-logarithmic approximation only two gluons in light-cone gauge connect the photon-vector meson system to the nucleus, as illustrated in Fig. 2. Thus, as predicted by PQCD color transparency $[18,11]$ the outgoing vector meson in effect suffers no final-state absorption, and the nuclear dependence of the $\gamma^{*} A \rightarrow V A$ forward amplitude will be identical to that for the case where the final state system is a virtual photon, $\gamma^{*} A \rightarrow \gamma^{*} A$; i.e. it will be close to additive in the nucleon number $A$. We can also understand this remarkable feature of QCD from the space-time arguments given above: the final state vector meson is formed from the compact $q \bar{q}$ pair over a long time $\tau_{f}$, and thus it does not attain its final physical size and its normal strong interactions until 
it is well outside the domain of the target nucleus. In fact, much of this physics was anticipated before the advent of QCD. The possibility that the outgoing absorption of the $\rho$ in the nucleus would be effectively small in large $Q^{2}$ leptoproduction was actually first proposed by Yennie in 1975 [19]. The observation that the incoming photon has point-like behavior and diminished absorption was discussed in terms of a "shrinking photon" by Cheng and Wu [20] and by Bjorken, Kogut, and Soper $[21]$.

We emphasize that the above reasoning is applicable for a longitudinally polarized vector meson only - the effective transverse size of a produced transversely polarized vector meson is considerably larger (although still smaller than for ordinary hadrons, $c f$. the discussion in Section 2.6).

Although the vector meson suffers no final state interactions, the forward amplitude $\mathcal{M}\left(\gamma^{*} A \rightarrow V A\right)$ is not strictly additive in nuclear number since the gluon distribution itself is shadowed. (This effect is similar to the shadowing of diffractive production of high- $p_{T}$ jets in the $\pi+A \rightarrow 2$ jets $+A$ reaction, discussed in Ref. [8].) In fact, we see from Eq. (2.33) that

$$
\left.\frac{d \sigma}{d t}\left(\gamma^{*} A \rightarrow V A\right)\right|_{t=0} \propto \alpha_{s}^{2}(Q)\left[x G_{A}(x, Q)\right]^{2}
$$

where $G_{A}(x, Q)=A^{\alpha_{g}(x, Q)} G_{N}(x, Q)$ is the gluon distribution in the nucleus. Thus, the analysis presented in this paper predicts identical nuclear dependence for the forward vector meson diffractive leptoproduction cross sections, the longitudinal structure functions $F_{A}^{L}(x, Q)$, and the square of the gluon structure functions:

$$
\frac{\left.\frac{d \sigma}{d t}\left(\gamma^{*} A \rightarrow V A\right)\right|_{t=0}}{\left.\frac{d \sigma}{d t}\left(\gamma^{*} N \rightarrow V N\right)\right|_{t=0}}=\left[\frac{F_{A}^{L}(x, Q)}{F_{N}^{L}(x, Q)}\right]^{2}=\frac{G_{A}^{2}(x, Q)}{G_{N}^{2}(x, Q)}=A^{2 \alpha_{g}(x, Q)}
$$

(Note that at finite energies one has to interpolate the cross section to the unphysi- 
cal $t=0$ kinematical point.) The nuclear gluon distribution is expected to be more strongly shadowed than the nuclear quark structure functions at intermediate $Q^{2}$ because of the larger color charge of the gluon in QCD and thus its stronger internuclear interactions. Numerical estimates [22] lead to $x G_{A}\left(x, Q_{0}^{2}\right) / A x G_{N}\left(x, Q_{0}^{2}\right) \sim$ $0.7-0.8(0.4-0.5)$ for $A=12(200)$ and $x \sim 0.01-0.03$, a result which seems to be supported by the recent FNAL data of E-665 [23]. However, at fixed $x \sim 0.01-0.03$, shadowing substantially decreases with $Q^{2}$ due to scaling violation effects [22], which should lead to an effective increase of transparency for $\rho$ leptoproduction at fixed $x$ with increasing $Q$.

A nuclear dependence similar to Eq. (2.38) is also expected for forward diffractive $\Upsilon$ and, possibly, $J / \psi$ leptoproduction cross sections even at small $Q^{2}$, although in this case the value of $x$ and the evolution scale of $G(x, Q)$ is controlled by $M_{V}^{2}$ rather than $Q^{2}$. Thus both heavy and light diffractive vector meson leptoproduction can provide basic information on the nature of the gluon distributions in nuclei.

The calculations given above are applicable to the near-forward production of vector mesons. It should be noted that the physics relevant to the nuclear dependence of the leptoproduction cross section will change with increasing $t$. We shall give here a semi-quantitative description of the expected behavior. At $-t R_{A}^{2} / 3 \ll 1$, coherent processes dominate the leptoproduction of vector mesons. The nuclear dependence of the diffractive cross section at small $t$ (within the diffractive peak) can be estimated by multiplying Eq. (2.38) by the square of electromagnetic form factor of nucleus normalized to 1 at $t=0$. However, if $-t R_{A}^{2} / 3 \gg 1$, incoherent processes, in which the leading vector meson is accompanied by the production of other hadrons from nuclear disintegration, will dominate the cross section. The existence of nuclear shadowing implies that gluons at small $x$ cannot 
be associated with individual nucleons. Thus one can have events where momenta $-t \geq 0.1 \mathrm{GeV}^{2}$ are transferred to each of several nucleons which subsequently fragment. The effect can be a slower $t$-dependence of the cross section and a smaller energy transfer per interacting target nucleon than for the scattering off a single nucleon. The expected $A$-dependence is intermediate between that expected for shadowing of $G_{A}\left(x, Q^{2}\right)$ and $A$.

The recent nuclear target $\rho$ leptoproduction measurements from the E-665 experiment [16] appear to indicate onset of the color transparency predicted by PQCD for incoherent $\gamma^{*} A \rightarrow \rho N(A-1)^{\prime}$ reactions. The onset of this phenomena is again for $Q^{2} \simeq$ a few $\mathrm{GeV}^{2}$, the same scale at which Bjorken scaling is observed in deep inelastic lepton scattering reactions. Preliminary data [15] from the NMC also confirm higher values of the transparency ratio for $Q^{2} \geq 3 \mathrm{GeV}^{2}$, observed in [16] although the NMC data do not indicate a $Q^{2}$ variation of transparency in their $10 \geq Q^{2} \geq 3 \mathrm{GeV}^{2}$ range. One needs to be cautious in interpreting these data directly in terms of PQCD color transparency of the outgoing $\rho$. We note that the high- $Q^{2}$ NMC data correspond to a range of $x$ where the essential longitudinal distances are smaller than the nucleus size. Thus transparency in this range of kinematics will reflect to some extent the fact that the virtual photon can penetrate deeper into nucleus without interaction. As we have emphasized, the nuclear dependence of forward diffractive $\rho$ leptoproduction which is completely coherent on the nucleus can provide a decisive test of color transparency. 


\section{Conclusions}

The analysis of diffractive leptoproduction cross sections presented in this paper extends the domain of PQCD predictions to a new domain of exclusive hadronic reactions. The central focus of this analysis is closely related to the calculations of the order $\alpha_{s}\left(Q^{2}\right)$ leading twist perturbative contributions to $\sigma_{L}\left(\nu, Q^{2}\right)$ and the violation of the Callan-Gross relation. Although the momentum transfer to the target is small, the virtuality of the longitudinally polarized photon provides a point-like probe of the diffractive $\gamma^{*} N \rightarrow V N$ process. At high $Q^{2}$ the amplitude factorizes in terms of separable components: the perturbative distribution amplitude of the virtual photon, the non-perturbative distribution amplitude of the outgoing vector meson system, and a non-perturbative two-gluon matrix element of the target closely related to the gluon structure function. The momentum transfer dependence of the diffractive amplitude is thus controlled by a new type of non-local two-gluon form factor. We note that since the momentum transfer to the nucleon is shared by two gluons, the fall-off can be different from the fall-off of elastic electromagnetic form factors.

The factorization analysis can be extended to the diffractive production of any vector meson system of mass $\mathcal{M}$ as long as $Q^{2}>\mathcal{M}^{2}$. The longitudinal cross section always falls as $1 / Q^{6}$ at fixed $\mathcal{M}, t$ and $s$. The energy dependence of the forward diffractive cross section is also universal, reflecting the behavior of the square of the gluon structure function at $x \sim Q^{2} / s$. Related formalisms have also been applied to exclusive heavy quarkonium photoproduction [4], exclusive pion dissociation to two jets [22], and to two-photon diffractive reactions [24].

The existing data for diffractive $\rho$ leptoproduction appear to be consistent

even at relatively low $Q^{2}$ of a few $\mathrm{GeV}^{2}$ with the leading logarithmic predictions in 
both magnitude and in the kinematic dependence. A crucial feature of the PQCD predictions, which is clearly evident in the data, is the increasing dominance of the longitudinal photon and vector meson production amplitudes with increasing $Q^{2}$. More careful studies of the longitudinal to transverse polarization ratios can lead to insights into the transition between soft and hard components in QCD amplitudes. As we have emphasized, precise measurements of the $A$ dependence of the diffractive leptoproduction reactions can lead to new insights into nuclear shadowing of the longitudinal structure function.

In our discussion we have noted only a few of the many empirical tests of PQCD possible in diffractive leptoproduction. In principle, the study of these reactions at HERA will allow tests of the theory over a huge dynamical range in $x$ and $Q^{2}$. The intercomparison of the various vector meson channels can also lead to new tests and understanding of the non-perturbative structure of hadronic wavefunctions and their flavor-symmetry properties.

\section{Acknowledgements}

We would like to thank Andrzej Sandacz of NMC and Guang Yin Fang of E-665 for illuminating discussions of the vector meson leptoproduction data. SJB, JFG and AM would also like to thank the Institute for Theoretical Physics, University of California, Santa Barbara, for support during the early stages of this project. 


\section{APPENDIX}

The $\gamma \rightarrow V$ transition is defined by

$$
\left\langle 0\left|J_{\mu}\right| V\right\rangle=\frac{\sqrt{2} e f_{V} \epsilon_{\mu}^{V}}{m_{V}}
$$

In the frame defined by Eq. (2.2) (where we shall take $Q^{2}=-m_{V}^{2}$ ), and employing the time-ordered formalism outlined in this paper, we find

$$
\frac{\sqrt{2} e f_{V} \epsilon^{\gamma} \cdot \epsilon^{V}}{m_{V}}=\sqrt{N_{c}} \sum_{\lambda_{1} \lambda_{2}} \int \frac{d^{2} k_{\perp} d^{2} k_{\perp}^{\prime}}{\left(16 \pi^{3}\right)^{2}} \int d z \int d z^{\prime} \psi_{\lambda_{1} \lambda_{2}}^{\gamma}\left(k_{\perp}, z\right) T \psi_{\lambda_{1} \lambda_{2}}^{V}\left(k_{\perp}^{\prime}, z^{\prime}\right)
$$

where the amplitude $T$ is trivial,

$$
T=16 \pi^{3} \delta\left(k_{\perp}^{\prime}-k_{\perp}\right) \delta\left(z^{\prime}-z\right)
$$

and $\psi^{\gamma}$ and $\psi^{V}$ are given by Eqs. (2.10) and (2.22), respectively. In the chosen frame, it will be most convenient to isolate $f_{V}$ by considering a longitudinally polarized $V$, with polarization vector as given in Eq. (2.5), while taking $\epsilon_{-}^{\gamma}=1$ and all other components of $\epsilon^{\gamma}$ to be 0 , implying that

$$
\epsilon^{\gamma} \cdot \epsilon^{V}=\frac{1}{2} \epsilon_{+}^{V}(L)=\frac{\frac{1}{2} q_{+}}{m_{V}}
$$

Meanwhile, in Eq. (2.10) $\gamma \cdot \epsilon^{\gamma}=\frac{1}{2} \gamma_{+}$while in Eq. (2.22), for $L$ polarization, $\gamma \cdot \epsilon^{V}$ can be approximated by $\frac{1}{2} \gamma_{+} \epsilon_{-}^{V}=-\frac{1}{2} \gamma_{+} m_{V} / q_{+}$. Using the fact that

$$
\frac{\bar{u}_{\lambda_{1}}(k)}{\sqrt{z}} \frac{1}{2} \gamma_{+} \frac{v_{\lambda_{2}}(q-k)}{\sqrt{1-z}}=\frac{\bar{v}_{\lambda_{2}}(q-k)}{\sqrt{1-z}} \frac{1}{2} \gamma_{+} \frac{u_{\lambda_{1}}(k)}{\sqrt{z}}=q_{+} \delta_{\lambda_{1}-\lambda_{2}}
$$


we obtain from Eq. (A.2) the result (with $Q^{2}=-m_{V}^{2}$ in (2.10)):

$$
\frac{\sqrt{2} e f_{V}\left(\frac{1}{2} q_{+}\right)}{m_{V}^{2}}=\sqrt{N_{c}} e e_{f} N_{V}\left(q_{+}^{2}\right) \sum_{\lambda} \int \frac{d^{2} k_{\perp}}{16 \pi^{3}} \int d z \frac{\psi^{V}\left(k_{\perp}, z\right)}{m_{V}}\left[\frac{-m_{V} / q_{+}}{-m_{V}^{2}}\right]
$$

With $\sum_{\lambda}=2$ and using the definition Eq. (2.25), we obtain

$$
\int_{0}^{1} d z \phi^{V}(z)=\frac{f_{V}}{\sqrt{N_{c}} e_{f} 2 \sqrt{2} N_{V}}
$$




\section{References}

1. V. N. Gribov, B. L. Ioffe, and I. Ya. Pomeranchuk, Yad. Fiz., 2, 768 (1965); B. L. Ioffe, Phys. Lett. 30123 (1968).

2. A. Donnachie and P. Landshoff, Phys. Lett. 185B, 403 (1987); Nucl. Phys. B311, 509 (1989).

3. J. R. Cudell, Nucl. Phys. B336, 1 (1990).

4. M. G. Ryskin, Z. Phys. C57, 89-92 (1993).

5. S. J. Brodsky and G. P. Lepage, Phys. Rev. D22, 2157 (1980).

6. B. Z. Kopeliovich, J. Nemchick, N. N. Nikolaev, and B. G. Zakharov, KFAIKP-TH-1993-27, (1993).

7. B. Blättel, G. Baym, L. L. Frankfurt, and M. Strikman, Phys. Rev. Lett. 71, 896 (1993).

8. L. Frankfurt, G. A. Miller, and M. Strikman, Phys. Lett. 304B, 1 (1993).

9. S. Catani, M. Ciafaloni, and F. Hautmann, Phys. Lett. B307, 147-153 (1993).

10. J. D. Bjorken in Proceedings of the International Symposium on Electron and Photon Interactions at High Energies, p. 281-297, Cornell (1971); J. D. Bjorken and J. B. Kogut, Phys. Rev. D8, 1341 (1973).

11. L. Frankfurt and M. Strikman, Phys. Rept. 160, 235 (1988).

12. V. L. Chernyak and A. R. Zhitnitski, Phys. Rep. 112, 173 (1984).

13. EMC, J. J. Aubert et al., Phys. Lett. 161B, 203 (1985); J. Ashman et al., Z. Phys. C39, 169 (1988).

14. NMC, P. Amaudruz et al., Z. Phys. C54, 239 (1992). 
15. NMC Collaboration, A. Sandacz, Exclusive $\rho^{0}$ Muoproduction at Large $Q^{2}$, paper contributed to the poster session at the Few Body XIV Conference, Amsterdam, 1993.

16. Guang Yin Fang, et al., FERMILAB-CONF-93-305, October 1993.

17. A. D. Martin, W. J. Stirling, and R. G. Roberts, Phys. Lett. B306, 145 (1993).

18. S. J. Brodsky and A. H. Mueller, Phys. Lett. 206B, 685 (1988).

19. D. R. Yennie, Rev. Mod. Phys. 47, 311 (1975). T. H. Bauer et al., Rev. Mod. Phys. 50, 261 (1978).

20. H. Cheng and T. T. Wu, Phys. Rev. 183, 1324 (1969).

21. J. Bjorken, J. Kogut, and D. Soper, Phys. Rev. D3, 1382 (1971).

22. L. Frankfurt, M. Strikman, and S. Liuti, Phys. Rev. Lett. 65, 1725 (1990); Proceeding of PANIC 93 (in press).

23. Harry L. Melanson, et al., FERMILAB-CONF-93-165-E, Presented at the 28th Rencontres de Moriond: QCD and High Energy Hadronic Interactions, Les Arcs, France, 20-27 March 1993.

24. I. F. Ginzburg and D. Yu. Ivanov, Nucl. Phys. B388, 376 (1992), and references therein. 


\section{FIGURE CAPTIONS}

1) A typical two-gluon exchange contribution to the amplitude for $\gamma^{*}(q) p \rightarrow$ $V(q+\Delta)(p-\Delta)$.

2) Light-cone perturbation theory graphs for the scattering of a quark-antiquark pair by a colorless target. 
This figure "fig1-1.png" is available in "png" format from: http://arxiv.org/ps/hep-ph/9402283v2 
This figure "fig1-2.png" is available in "png" format from: http://arxiv.org/ps/hep-ph/9402283v2 